\title{
Group Analysis of Free Convection Flow of a Magnetic Nanofluid with Chemical Reaction
}

\author{
Md. Jashim Uddin, ${ }^{1}$ O. A. Bég, ${ }^{2}$ A. Aziz, ${ }^{3}$ and A. I. Md. Ismail ${ }^{1}$ \\ ${ }^{1}$ School of Mathematical Sciences, Universiti Sains Malaysia (USM), 11800 Penang, Malaysia \\ ${ }^{2}$ Gort Engovation Research (Propulsion, Nanofluids and Biomechanics), Bradford BD73NU, UK \\ ${ }^{3}$ School of Engineering and Applied Science, Gonzaga University, E. 502 Boone Avenue, Spokane, WA 99258, USA \\ Correspondence should be addressed to Md. Jashim Uddin; jashim_74@yahoo.com
}

Received 22 September 2014; Revised 8 January 2015; Accepted 16 January 2015

Academic Editor: Hang Xu

Copyright (C) $2015 \mathrm{Md}$. Jashim Uddin et al. This is an open access article distributed under the Creative Commons Attribution License, which permits unrestricted use, distribution, and reproduction in any medium, provided the original work is properly cited.

\begin{abstract}
A theoretical study of two-dimensional magnetohydrodynamics viscous incompressible free convective boundary layer flow of an electrically conducting, chemically reacting nanofluid from a convectively heated permeable vertical surface is presented. Scaling group of transformations is used in the governing equations and the boundary conditions to determine absolute invariants. A thirdorder ordinary differential equation which corresponds to momentum conservation and two second-order ordinary differential equations which correspond to energy and nanoparticle volume fraction (species) conservation are derived. Our (group) analysis indicates that, for the similarity solution, the convective heat transfer coefficient and mass transfer velocity are proportional to $x^{-1 / 4}$ whilst the reaction rate is proportional to $x^{-1 / 2}$, where $x$ is the axial distance from the leading edge of the plate. The effects of the relevant controlling parameters on the dimensionless velocity, temperature, and nanoparticle volume fraction are examined. The accuracy of the technique we have used was tested by performing comparisons with the results of published work and the results were found to be in good agreement. The present computations indicate that the flow is accelerated and temperature enhanced whereas nanoparticle volume fractions are decreased with increasing order of chemical reaction. Furthermore the flow is strongly decelerated, whereas the nanoparticle volume fraction and temperature are enhanced with increasing magnetic field parameter. Increasing convection-conduction parameter increases velocity and temperatures but has a weak influence on nanoparticle volume fraction distribution. The present study demonstrates the thermal enhancement achieved with nanofluids and also magnetic fields and is of relevance to nanomaterials processing.
\end{abstract}

\section{Introduction}

Nanofluids have a significant impact on heat transfer enhancement in modern years. They have been utilized in diverse technologies including turbulent flows [1], propulsion [2], geothermics [3], porous media solar collectors $[4,5]$, and chemical engineering coating processes [6]. Further, interesting implementations of nanofluids include rocket propellant combustion [7], drug delivery and food biophysics [8], crystal growth [9], polymeric materials processing [10], and automotive engine cooling [11]. Nanofluids constitute a significant class of heat transfer fluids obtained by dispersing certain nanoparticles ( $<100$ nanometers in diameter) in conventional poor thermal conductivity base fluids. Nanofluids aim to achieve the maximum possible thermal properties with the minimum possible concentration by uniform dispersion and stable suspension of nanoparticles in host fluids $[12,13]$. Nanofluids are capable of enhancing thermophysical properties such as thermal conductivity, thermal diffusivity, viscosity, and convective heat transfer coefficients compared to those of the base fluids like water, ethylene or triethyleneglucose and other coolants, biofluids, and polymer solutions, as elaborated by Choi [14] and Wong and Leon [15]. In parallel with this, a subbranch of nanofluids termed magnetic nanofluids has also shown significant promise in numerous engineering fields. These fluids respond to applied magnetic fields and allow further manipulation of heat transfer and hydrodynamic characteristics. Often magnetite 
and aluminum oxide nanoparticles are exploited in the architecture of such fluids. Important experimental studies of magnetic nanofluids have been conducted by Parekh and Lee [16]. The tribological performance of magnetic nanofluids has been recently elucidated by Andablo-Reyes et al. [17]. Thermal enhancement features of magnetic nanofluids have also been demonstrated by Chiang et al. [18]. Biomedical uses for magnetic nanofluids have been confirmed by Patel [19]. Numerical simulations of magnetic nanofluids have been reported by Rana et al. [20] for nanomaterials processing operation using a finite element method. Very recently, Yadav et al. [21] studied the magnetohydrodynamic stability of magnetic nanofluids using a numerical Galerkin technique for the case of magnetite alumina-water systems.

Mathematical modelling of nanofluids was greatly simplified by the seminal study of Buongiorno [22] who clarified that the key mechanisms contributing to thermal enhancement are Brownian diffusion and thermophoresis. Building on this, an important boundary layer model for free convective flows of nanofluids was first presented by Kuznetsov and Nield [23] which laid the foundation for many subsequent studies. Magnetohydrodynamic boundary layer nanofluid flow was later investigated by Hamad et al. [24]. Chamkha and Aly [25] further studied magneto-nanofluid flow using the Blottner implicit difference method and also considered heat generation effects.

Generally, most studies involving magnetohydrodynamics and nanofluids assume either constant wall temperature or constant heat flux at the surface. Aziz [26] introduced the idea of using the convective heating boundary condition to analyze Blasius flow. This condition was shown to exert a significant influence on flow characteristics in the boundary layer. Subsequently, the convective boundary condition was utilized by a variety of researchers including Yao et al. [27] for stretching/shrinking flows, Makinde and Aziz [28] for nanofluids, Yacob et al. [29] for nanofluids, Uddin et al. [30] for nanofluid flow in porous media, and Aziz and Khan [31] for natural convective nanofluid.

These studies have neglected chemical reaction effects. Reaction is an important mechanism in materials processing operations. Chemical reactions may be homogenous, heterogenous, exothermic, or endothermic. They may be of first order or higher. Numerous boundary layer convection simulations in the presence of chemical reaction have been communicated in many diverse areas including combustion [32], geophysics [33], polymer melt flows [34], geothermics [35], PEM fuel cells [36], and solar collectors [37]. Makinde et al. [38] studied chemical reaction and cross-diffusion effects on magnetic heat transfer using the Aziz convective surface boundary condition, showing that increasing order of chemical reaction increases concentration boundary layer thickness whereas species diffusion is opposed with increasing destructive chemical reaction rate. Furthermore, this study showed that thermal boundary layer thickness was enhanced with increasing local convective heat transfer parameter and destructive chemical reaction rate but depressed with increasing chemical reaction order. Chemical reactions are important also in nanofluid systems, although very little work has been reported concerning theoretical studies. Chen et al. [39] have provided a detailed exposition of nanofluids designed using specific chemical reactions. Ismoen et al. [40] have studied the nanofluid convection with suction effects, chemical reaction, and thermal radiation. Kameswaran et al. [41] have used MATLAB to model the convective heat and mass transfer in dissipative and reactive magneto-nanofluid flow with Soret diffusion effects for the cases of copper-water and silver-water nanofluids. Rosca et al. [42] have studied reactive nanofluid stagnationpoint flow. These studies used a relatively simple nanofluid model developed by Tiwari and Das [43]. In the current study we extend the recent work of Aziz and Khan [31] to include higher order chemical reaction, magnetic field, and suction/injection effects. A more elaborate nanofluid transport model is employed than discussed previously. A one parameter group method is utilized to develop similarity transformations for the governing conservation equations and boundary conditions. The resulting nonlinear boundary value problem is solved with MAPLE 17 numerical quadrature. The influences of chemical reaction, magnetic field, wall transpiration, convective-conductive boundary condition, Brownian parameter, thermophoresis parameter and Lewis number on the dimensionless fluid velocity, temperature, and nanoparticle volume fraction distributions are explored. Validation of the solutions with earlier nonreactive nanofluid studies is also included.

The investigations of problems related to heat and nanoparticle volume fraction transfer with chemical reaction are of great practical importance to engineers and scientists due to their occurrence in many branches of science and engineering. A few representative fields of interest where combined heat and nanoparticle volume fraction transfer along with chemical reaction play an important role are in chemical process industries such as food processing and polymer production. A good area of applications of the present problem is nanofluid synthesis for medicine.

\section{Mathematical Transport Model for Reactive Magneto-Nanofluid Convection}

We examine the two-dimensional, steady magnetohydrodynamics (MHD) free convective nanofluid boundary layer flow over a permeable flat vertical plate as shown in Figure 1 (i, ii, and iii represent momentum and thermal and nanoparticle volume fraction boundary layers, resp.). We consider the case of a variable magnetic field of strength $B(\bar{x})$ applied normal to the plate (in order to get true similarity solution). It is assumed that the electric conductivity $\sigma$ is variable. Following Samanta and Guha [44] and Uddin et al. [45], we assumed that $\sigma=\sigma_{0} \bar{u}, \sigma_{0}$ is the constant electric conductivity and $B(\bar{x})=B_{0} / \sqrt{\bar{x}}, B_{0}$ is the constant magnetic field strength.

One can anticipate the fact that when $B(\bar{x})=B_{0} / \sqrt{\bar{x}}$, there is a singular point at $\bar{x}=0$ (i.e., at the leading edge). However, it is well known that the boundary layer equations are not valid there.

A homogeneous isothermal irreversible chemical reaction of order $n$ is deemed to take place between the surface of the plate and fluid. We further assume the left surface 


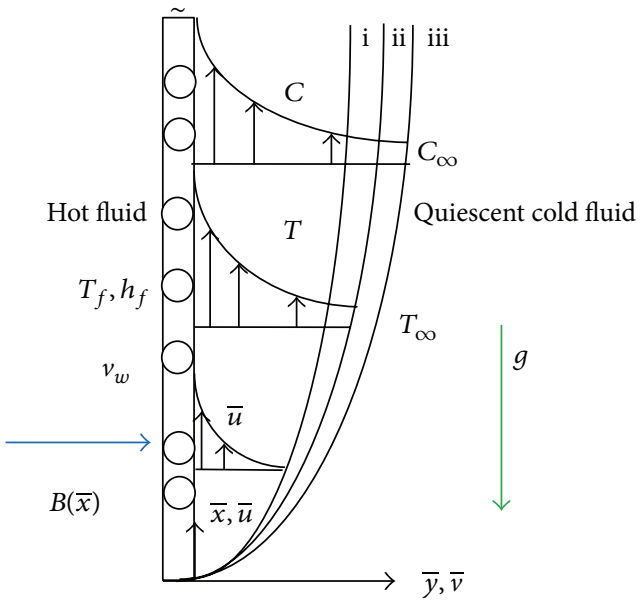

FIGURE 1: Flow configuration and coordinate system.

of the plate to be heated by convection from a hot fluid at a temperature $T_{f}(\bar{x})$ with a heat transfer coefficient $h_{f}(\bar{x})$. The temperature of the right surface of the plate is denoted by $T_{w}$ where $T_{w}>T_{\infty}$. The nanoparticle volume fraction of the wall is denoted by $C_{w}$ whilst the ambient values of the temperature and the nanoparticle volume fraction are denoted, respectively, by $T_{\infty}$ and $C_{\infty}$. We assume that (i) the electric conductivity and reaction rate order are variable, (ii) density of the fluid is constant except the body force term, (iii) the concentration of nanoparticles is sufficiently diluted that particle coagulation in the boundary layer can be neglected, (iv) the magnetic Reynolds number is sufficiently small that the induced magnetic field can be neglected when compared to the applied magnetic field, (v) the Hall effect, viscous dissipation, and Joule heating terms are neglected, and (vi) the external electric field as well as the electric field due to the polarization of charges can be ignored. For the unsteady and incompressible nanofluids, the following four equations embodying the conservation of mass, momentum, thermal energy, and nanoparticle volume fraction in the vectorial form are (Buongiorno [22], Kuznetsov and Nield [23])

$$
\nabla \cdot \mathbf{v}=0
$$

$$
\begin{aligned}
& \rho\left(\frac{\partial \mathbf{v}}{\partial t}+\mathbf{v} \cdot \nabla \mathbf{v}\right) \\
& =-\nabla p+\mu \nabla^{2} \mathbf{v} \\
& +\left[\phi \rho_{p}+(1-\phi) \rho_{f}\left\{\beta\left(T-T_{\infty}\right)\right\}\right] \mathbf{g}+\mathbf{J} \times \mathbf{B}, \\
& \frac{\partial T}{\partial t}+\mathbf{v} \cdot \nabla T=\alpha \nabla^{2} T \\
& +\tau\left[D_{B} \nabla T \cdot \nabla C+\left(\frac{D_{T}}{T_{\infty}}\right) \nabla T \cdot \nabla T\right], \\
& \frac{\partial C}{\partial t}+\mathbf{v} \cdot \nabla C=D_{B} \nabla^{2} C+\left(\frac{D_{T}}{T_{\infty}}\right) \nabla^{2} T \\
& +k\left(C-C_{\infty}\right)^{n} \text {, }
\end{aligned}
$$

where $\mathbf{v}$ is the velocity vector, $T$ is the temperature, $C$ is the nanoparticle volume fraction, $p$ is the pressure, $v$ is the kinematic viscosity, $\rho$ is the fluid density, $D_{B}$ is the Brownian diffusion coefficient and $D_{T}$ is the thermophoretic diffusion coefficient, $\mathbf{J}=\sigma(\mathbf{E}+\mathbf{V} \times \mathbf{B})$ is the electric current density, $\mathbf{B}$ is the magnetic induction vector, and $\mathbf{E}$ is the electric field. We assume that electric filed neglect $\mathbf{E}=0$. It is assumed that $\mathbf{B}=(0, B, 0)$. We consider steady-state flow and we write $\mathbf{v}=(\bar{u}, \bar{v})$. The Oberbeck-Boussinesq approximation is used and with these assumptions and the standard boundary layer assumptions, we obtain the governing equations in dimensional form as follows:

$$
\begin{gathered}
\frac{\partial \bar{u}}{\partial \bar{x}}+\frac{\partial \bar{v}}{\partial \bar{y}}=0 \\
\frac{\partial p}{\partial \bar{x}}=\mu \frac{\partial^{2} \bar{u}}{\partial \bar{y}^{2}}-\frac{1}{\rho_{f}} \sigma_{0} B^{2}(\bar{x}) \bar{u}^{2}-\rho_{f}\left(\bar{u} \frac{\partial \bar{u}}{\partial \bar{x}}+\bar{v} \frac{\partial \bar{u}}{\partial \bar{y}}\right) \\
+\left[\left(1-C_{\infty}\right) \rho_{f_{\infty}} \beta g \beta\left(T-T_{\infty}\right)\right. \\
\left.-\left(\rho_{p}-\rho_{f_{\infty}}\right) g\left(C-C_{\infty}\right)\right], \\
\bar{u} \frac{\partial T}{\partial \bar{x}}+\bar{v} \frac{\partial T}{\partial \bar{y}}=\alpha \frac{\partial^{2} T}{\partial \bar{y}^{2}}+\tau\left[D_{B} \frac{\partial C}{\partial \bar{y}} \frac{\partial T}{\partial \bar{y}}+\frac{D_{T}}{T_{\infty}}\left(\frac{\partial T}{\partial \bar{y}}\right)^{2}\right], \\
\bar{u} \frac{\partial C}{\partial \bar{x}}+\bar{v} \frac{\partial C}{\partial \bar{y}}=D_{B} \frac{\partial^{2} C}{\partial \bar{y}^{2}}+\frac{D_{T}}{T_{\infty}} \frac{\partial^{2} T}{\partial \bar{y}^{2}}-k(\bar{x})\left(C-C_{\infty}\right)^{n} .
\end{gathered}
$$

This model extends the earlier model of Aziz and Khan [31] with the inclusion of magnetohydrodynamic body force, chemical reaction, and wall transpiration effect.

The boundary conditions are

$$
\begin{array}{r}
\bar{u}=0, \quad \bar{v}=v_{w}(\bar{x}), \quad-k \frac{\partial T}{\partial \bar{y}}=h_{f}(\bar{x})\left(T_{f}-T_{w}\right), \quad C=C_{w} \\
\text { at } \bar{y}=0,
\end{array}
$$

$\bar{u} \longrightarrow 0, \quad T \longrightarrow T_{\infty}, \quad C \longrightarrow C_{\infty} \quad$ as $\bar{y} \longrightarrow \infty$,

where $(\bar{u}, \bar{v})$ is velocity components along and perpendicular directions of the plate, $v_{w}(\bar{x})$ is suction/injection velocity, $\tau=(\rho c)_{p} /(\rho c)_{f}$ is ratio of nanoparticle heat capacity and the base fluid heat capacity, $\alpha=k /(\rho c)_{f}$ is thermal diffusivity of the fluid, $\rho_{f}$ is density of the base fluid, $\sigma$ is variable electric conductivity, $\mu, k$, and $\beta$ denote viscosity, thermal conductivity, and volumetric expansion coefficient of the nanofluid and $\rho_{p}$ represents density of the particles, $\mathrm{g}$ is acceleration due to gravity, $D_{B}$ is Brownian diffusion coefficient, and $D_{T}$ is thermophoretic diffusion coefficient. It is convenient for the solution of our problem, to express (2)-(6) in dimensionless form. Thus, we define the following dimensionless quantities:

$$
\begin{aligned}
& x=\frac{\bar{x}}{L}, \quad y=\frac{\bar{y} \mathrm{Ra}^{1 / 4}}{L}, \quad u=\frac{\bar{u} L}{\alpha \mathrm{Ra}^{1 / 2}}, \\
& v=\frac{\bar{v} L}{\alpha \mathrm{Ra}^{1 / 4}}, \quad \theta=\frac{T-T_{\infty}}{T_{f}-T_{\infty}}, \quad \phi=\frac{C-C_{\infty}}{C_{w}-C_{\infty}} .
\end{aligned}
$$


Here $L$ is a characteristic length for the vertical surface and $\mathrm{Ra}=\left(1-C_{\infty}\right) g \beta \rho_{f_{\infty}} \Delta T L^{3} / \alpha \nu$ is the Rayleigh number. A dimensional stream function $\psi$ defined as $u=\partial \psi / \partial y, v=$ $-\partial \psi / \partial x$ is introduced into (3)-(6) so as to reduce the number of equations as well as number of dependent variables. We then obtain the following equations:

$$
\begin{gathered}
\frac{\partial \psi}{\partial y} \frac{\partial^{2} \psi}{\partial x \partial y}-\frac{\partial \psi}{\partial x} \frac{\partial^{2} \psi}{\partial y^{2}}-\operatorname{Pr} \frac{\partial^{3} \psi}{\partial y^{3}} \\
+\frac{M}{x}\left(\frac{\partial \psi}{\partial y}\right)^{2}-\operatorname{Pr}[\theta-\mathrm{Nr} \phi]=0 \\
\frac{\partial \psi}{\partial y} \frac{\partial \theta}{\partial x}-\frac{\partial \psi}{\partial x} \frac{\partial \theta}{\partial y}-\frac{\partial^{2} \theta}{\partial y^{2}} \\
-\mathrm{Nb} \frac{\partial \theta}{\partial y} \frac{\partial \phi}{\partial y}-\mathrm{Nt}\left(\frac{\partial \theta}{\partial y}\right)^{2}=0 \\
\frac{\partial \psi}{\partial y} \frac{\partial \phi}{\partial x}-\frac{\partial \psi}{\partial x} \frac{\partial \phi}{\partial y}-\frac{1}{\operatorname{Le}} \frac{\partial^{2} \phi}{\partial y^{2}} \\
-\frac{\mathrm{Nt}}{\mathrm{Nb}} \frac{1}{\mathrm{Le}} \frac{\partial^{2} \theta}{\partial y^{2}}+\frac{L(\Delta C)^{n-1}}{\alpha \sqrt{\mathrm{Ra}}} k(x) \phi^{n}=0
\end{gathered}
$$

subject to the nondimensional boundary conditions:

$$
\begin{aligned}
& \frac{\partial \psi}{\partial y}=0, \quad \frac{\partial \psi}{\partial x}=\frac{L}{\alpha \mathrm{Ra}^{1 / 4}} v_{w}(x), \\
& \frac{\partial \theta}{\partial y}=-\frac{L h_{f}(x)}{k \mathrm{Ra}^{1 / 4}}(1-\theta), \quad \phi=1 \\
& \quad \text { at } y=0, \\
& \frac{\partial \psi}{\partial y} \longrightarrow 0, \quad \theta \longrightarrow 0, \quad \phi \longrightarrow 0 \quad \text { as } y \longrightarrow \infty
\end{aligned}
$$

Here the following definitions apply: $M=\sigma_{0} B_{0}^{2} / \rho_{f}$ (magnetohydrodynamic parameter), $\operatorname{Pr}=\nu / \alpha$ (Prandtl number), $\mathrm{Nt}=\tau D_{T} \Delta T / \alpha T_{\infty}$ (thermophoresis), $\mathrm{Nb}=\tau D_{B} \Delta C / \alpha$ (Brownian motion), $\mathrm{Nr}=\left(\rho_{p}-\rho_{f_{\infty}}\right) \Delta C / \rho_{f_{\infty}} \beta \Delta T\left(1-C_{\infty}\right)$ (buoyancy ratio), and Le $=\alpha / D_{B}$ (Lewis number).

Group theoretic methods are a powerful, sophisticated, and systematic technique which allows the derivation of similarity equations. Using the group method, we can obtain existing similarity solutions as well as new similarity solutions [45]. Group methods reduce the number of independent variables with one or more of the governing system of partial differential equations keeping the system and auxiliary conditions invariant. The method combines the independent variables into a single independent variable (which is known as the similarity variable) $[47,48]$. The original initial and boundary conditions become two boundary conditions in the new combined variable [49]. The main benefit of this method is that it can be successfully applied to nonlinear differential equations. The technique has been successfully utilized in non-Newtonian flows [50] turbulence [51], chemically reacting flows [52], cross-diffusion flows [53], stagnation flows in porous media [54], variable-viscosity convection [55], magnetic nanofluid flows [56], and radiative flows [5759]. Group theoretic methods have also been reviewed in the context of subsonic magnetofluid dynamics by Bég [60]. A simplified form of Lie-group transformations, namely, the scaling group of transformations, is used to determine the invariant solutions (similarity solutions) of (8)-(10) and boundary conditions (11):

$$
\begin{gathered}
\Gamma: x^{*}=x e^{\varepsilon \alpha_{1}}, \quad y^{*}=y e^{\varepsilon \alpha_{2}}, \quad \psi^{*}=\psi e^{\varepsilon \alpha_{3}}, \\
\theta^{*}=\theta e^{\varepsilon \alpha_{4}}, \quad \phi^{*}=\phi e^{\varepsilon \alpha_{5}}, \quad h_{f}^{*}=h_{f} e^{\varepsilon \alpha_{6}}, \\
k^{*}=k e^{\varepsilon \alpha_{7}}, \quad v_{w}^{*}=v_{w} e^{\varepsilon \alpha_{8}} .
\end{gathered}
$$

$\mathcal{E}$ is an arbitrary constant which is known as the parameter of the group and $\alpha_{i}(i=1,2, \ldots, 8)$ are arbitrary real numbers not all zero whose relationship will be determined later. The transformations in (11) can be thought of as a point transformation which transforms the coordinates $\left(x, y, \psi, \theta, \phi, h_{f}, k, v_{w}\right)$ to $\left(x^{*}, y^{*}, \psi^{*}, \theta^{*}, \phi^{*}, h_{f}{ }^{*}, k^{*}, v_{w}{ }^{*}\right)$. Substituting (12) into (8)-(11) and applying invariant condition led to

$$
\begin{array}{lll}
\alpha_{1}=4 \alpha_{2}, & \alpha_{3}=3 \alpha_{2}, & \alpha_{4}=\alpha_{5}=0, \\
\alpha_{6}=-\alpha_{2}, & \alpha_{7}=-2 \alpha_{2}, & \alpha_{8}=-\alpha_{2} .
\end{array}
$$

Expanding each transformation in (12) in Taylor's series in powers of $\varepsilon$, keeping the terms up to the order $\varepsilon$, and making use of the relation in (13) we obtain

$$
\begin{gathered}
x^{*}-x=\varepsilon 4 \alpha_{2} x, \quad y^{*}-y=\varepsilon \alpha_{2} y, \\
\psi^{*}-\psi=3 \varepsilon \alpha_{2} \psi, \quad \theta^{*}-\theta=0, \quad \phi^{*}-\phi=0, \\
h_{f}{ }^{*}-h_{f}=-\varepsilon \alpha_{2} h_{f}, \quad k^{*}-k=-2 \varepsilon \alpha_{2}, \\
v_{w}{ }^{*}-v_{w}=-\varepsilon \alpha_{2} v_{w} .
\end{gathered}
$$

We seek the absolute invariants under this group of transformations in (14). The absolute invariant is the function having the same form before and after the transformation. To obtain absolute invariants, we employ the following characteristic equations:

$$
\begin{aligned}
\frac{d x}{4 \alpha_{2} x} & =\frac{d y}{\alpha_{2} y}=\frac{d \psi}{3 \alpha_{2} \psi}=\frac{d \theta}{0}=\frac{d \phi}{0} \\
& =\frac{d h_{f}}{-\alpha_{2} h_{f}}=\frac{d k}{-2 \alpha_{2} k}=\frac{d v_{w}}{-\alpha_{2} v_{w}}, \quad \alpha_{2} \neq 0 .
\end{aligned}
$$

Solving (15) leads to the following transformations:

$$
\begin{aligned}
& \eta=\frac{y}{\sqrt[4]{x}}, \quad \psi=x^{3 / 4} f(\eta), \quad \theta=\theta(\eta), \quad \phi=\phi(\eta), \\
& h_{f}=\frac{h_{f_{0}}}{\sqrt[4]{x}}, \quad k=\frac{k_{0}}{\sqrt{x}}, \quad v_{w}=\frac{v_{w_{0}}}{\sqrt[4]{x}} .
\end{aligned}
$$

Here $\eta$ is the similarity independent variable and $f(\eta)$, $\theta(\eta)$, and $\phi(\eta)$ are, respectively, the dimensionless velocity, 
temperature, and nanoparticle volume fraction functions and $h_{f_{0}}, k_{0}$, and $v_{w_{0}}$ are constant heat transfer coefficient, reaction rate, and mass transfer velocity. With the use of the transformations in (16), (8)-(11) reduce to the following "similar" ordinary differential equations:

$$
\begin{gathered}
f^{\prime \prime \prime}+\frac{1}{4 \operatorname{Pr}}\left(3 f f^{\prime \prime}-2 f^{\prime 2}-4 M f^{2}\right)+\theta-\operatorname{Nr} \phi=0, \\
\theta^{\prime \prime}+\frac{3}{4} f \theta^{\prime}+\operatorname{Nb} \theta^{\prime} \phi^{\prime}+\mathrm{Nt} \theta^{\prime 2}=0, \\
\phi^{\prime \prime}+\frac{3}{4} \operatorname{Le} f \phi^{\prime}+\frac{\mathrm{Nt}}{\mathrm{Nb}} \theta^{\prime \prime}-\operatorname{Le} K \phi^{n}=0 .
\end{gathered}
$$

The appropriate prescribed boundary conditions assume

$$
\begin{aligned}
& f(0)=\frac{4}{3} f_{w}, \quad f^{\prime}(0)=0, \quad \theta^{\prime}(0)=-\gamma[1-\theta(0)], \\
& \phi(0)=1, \quad f^{\prime}(\infty)=\theta(\infty)=\phi(\infty)=0 .
\end{aligned}
$$

The emerging thermophysical parameters are defined as follows: $K=k_{0}(\Delta C)^{n-1} L / \alpha \sqrt{\mathrm{Ra}}$ (reaction), $f_{w}=$ $-v_{w_{0}} L \mathrm{Ra}^{1 / 4} / \alpha$ (suction/injection), and $\gamma=h_{f_{0}} L / \mathrm{Ra}^{1 / 4} k$ (thermal convective). Note that all parameters are free from axial distance $x$ which confirm the true similarity solution. The primes denote derivative with respect to $\eta$. It is pertinent to note that when $M=\mathrm{Nb}=\mathrm{Nt}=f_{w}=K=0$ and $\gamma \rightarrow \infty$, the present flow model is reduced to that considered by Bég [60]. Additionally for $M=f_{w}=K=0$ and $\gamma \rightarrow \infty$, our problem reduces exactly to the model studied by Kuznetsov and Nield [23]. Furthermore for $M=f_{w}=K=0$, our problem reduces to the equations of Aziz and Khan [31]. From an engineering perspective, several derivative quantities of the velocity, temperature, and nanoparticle concentration variables are of interest. The local Nusselt number $\mathrm{Nu}_{\bar{x}}$ and the Sherwood number $\mathrm{Sh}_{\bar{x}}$ are defined to be

$$
\mathrm{Nu}_{\bar{x}}=\frac{\bar{x} q_{w}^{\prime \prime}}{k(\Delta T)}, \quad \mathrm{Sh}_{\bar{x}}=\frac{\bar{x} q_{m}^{\prime \prime}}{D_{B}(\Delta C)},
$$

where $q_{w}^{\prime \prime}$ and $q_{m}^{\prime \prime}$ are the wall heat and mass fluxes, respectively, and $\tau_{w}$ is the wall shear stress. Following Kuznetsov and Nield [23], the reduced local Nusselt number $\mathrm{Nu}_{r}$ and reduced local Sherwood number $\mathrm{Sh}_{r}$ can be introduced and defined thus:

$$
\mathrm{Nu}_{r}=-\theta^{\prime}(0), \quad \mathrm{Sh}_{r}=-\phi^{\prime}(0) .
$$

\section{Numerical Solution with Maple 17}

Equations (17) subject to boundary conditions in (18) were solved numerically by using the Runge-Kutta-Fehlberg fourth-fifth order numerical method available in Maple 17. The validity of the present computations has been confirmed via benchmarking with several earlier studies. We have

\begin{tabular}{|c|c|c|c|c|}
\hline \multicolumn{3}{|c|}{ Present results } & \multicolumn{2}{|c|}{ Aziz and Khan [31] } \\
\hline \multirow{3}{*}{$\begin{array}{l}\mathrm{Nr} \\
0.1\end{array}$} & \multirow{3}{*}{$\begin{array}{l}\mathrm{Nu}_{r} \\
\quad \mathrm{Nb}=0.1 \\
\quad 0.39201\end{array}$} & \multirow{3}{*}{$\begin{array}{l}\mathrm{Sh}_{r} \\
\mathrm{Le}=10 \\
1.07969\end{array}$} & \multirow{2}{*}{\multicolumn{2}{|c|}{$\begin{array}{l}\mathrm{Nu}_{r} \quad \mathrm{Sh}_{r} \\
\mathrm{Nt}=0.1, \gamma=10, \mathrm{Pr}=10, \\
f_{w}=M=K=0\end{array}$}} \\
\hline & & & & \\
\hline & & & 0.3920 & 1.0797 \\
\hline 0.3 & 0.3850 & 1.0541 & 0.3850 & 1.0542 \\
\hline \multirow[t]{2}{*}{0.5} & 0.3776 & 1.026299 & 0.3775 & 1.0263 \\
\hline & $\mathrm{Nu}_{r}$ & $\mathrm{Sh}_{r}$ & $\mathrm{Nu}_{r}$ & $\mathrm{Sh}_{r}$ \\
\hline $\mathrm{Nb}$ & $\mathrm{Nr}=0.1$ & $\mathrm{Le}=10$ & \multicolumn{2}{|c|}{$\begin{array}{c}\mathrm{Nt}=0.1, \gamma=10, \operatorname{Pr}=10 \\
f_{w}=M=K=0\end{array}$} \\
\hline 0.3 & 0.34071 & 1.1295 & 0.3407 & 1.1295 \\
\hline 0.5 & 0.2944 & 1.1462 & 0.2944 & 1.1462 \\
\hline \multirow[b]{2}{*}{ Le } & $\mathrm{Nu}_{r}$ & $\mathrm{Sh}_{r}$ & $\mathrm{Nu}_{r}$ & $\mathrm{Sh}_{r}$ \\
\hline & $\mathrm{Nb}=0.1$ & $\mathrm{Nr}=0.1$ & \multicolumn{2}{|c|}{$\begin{array}{c}\mathrm{Nt}=0.1, \gamma=10, \operatorname{Pr}=10 \\
f_{w}=M=K=0\end{array}$} \\
\hline 1 & 0.39291 & 0.29277 & 0.3929 & 0.2928 \\
\hline 5 & 0.3934 & 0.7981 & 0.3934 & 0.7981 \\
\hline
\end{tabular}
compared our local skin friction factor represented by $f^{\prime \prime}(0)$ and the local reduced Nusselt number represented by $-\theta^{\prime}(0)$
TABLE 1: Comparison of our results for $f^{\prime \prime}(0)$ and $-\theta^{\prime}(0)$ with published results $(\mathrm{Le}=0.01)$.

\begin{tabular}{lcccc}
\hline & \multicolumn{2}{c}{$f^{\prime \prime}(0)$} & \multicolumn{2}{c}{$-\theta^{\prime}(0)$} \\
Pr & $\begin{array}{c}\text { Khair and } \\
\text { Bejan [46] }\end{array}$ & Present & $\begin{array}{c}\text { Khair and Bejan } \\
{[46]}\end{array}$ & $\begin{array}{c}\text { Present } \\
\text { results }\end{array}$ \\
\hline 0.01 & 0.44104 & 0.441699 & 0.1803 & 0.180194 \\
0.72 & 0.8806 & 0.880658 & 0.3871 & 0.387372 \\
10 & 1.0542 & 1.054089 & 0.4650 & 0.464912 \\
100 & 1.1428 & 1.124179 & 0.4898 & 0.488268 \\
1000 & 1.15205 & 1.144077 & 0.4985 & 0.495915 \\
\hline
\end{tabular}

TABLE 2: Comparison of our results for and with published results.

with the previously published data of Khair and Bejan [46]. The comparison is shown in Table 1 , and excellent agreement is achieved. Also we have compared our results with those of Aziz and Khan [31] and found a close agreement (Table 2). Confidence in the present results is therefore high. In the present computations, the asymptotic boundary conditions given in (18) were replaced by a finite value of 10 for similarity variable $\eta_{\max }$ as follows:

$$
\eta_{\max }=10, \quad f^{\prime}(10)=\theta(10)=\phi(10)=0 .
$$

The choice of $\eta_{\max }=10$ ensures that all numerical solutions approach the far field asymptotic values correctly. This is an important point which is sometimes overlooked. Pantokratoras [61] observed that many published results on the boundary layer flow are erroneous as the graphs for velocity, temperature, and concentration distributions in the boundary layers do not approach the correct values asymptotically. 


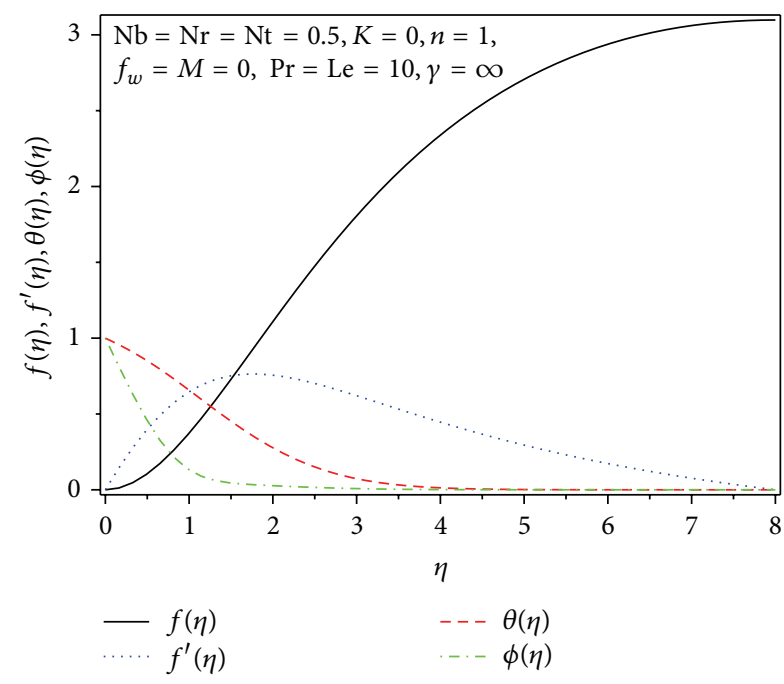

FIgURE 2: Thermofluid characteristics for Kuznetsov-Nield case [23].

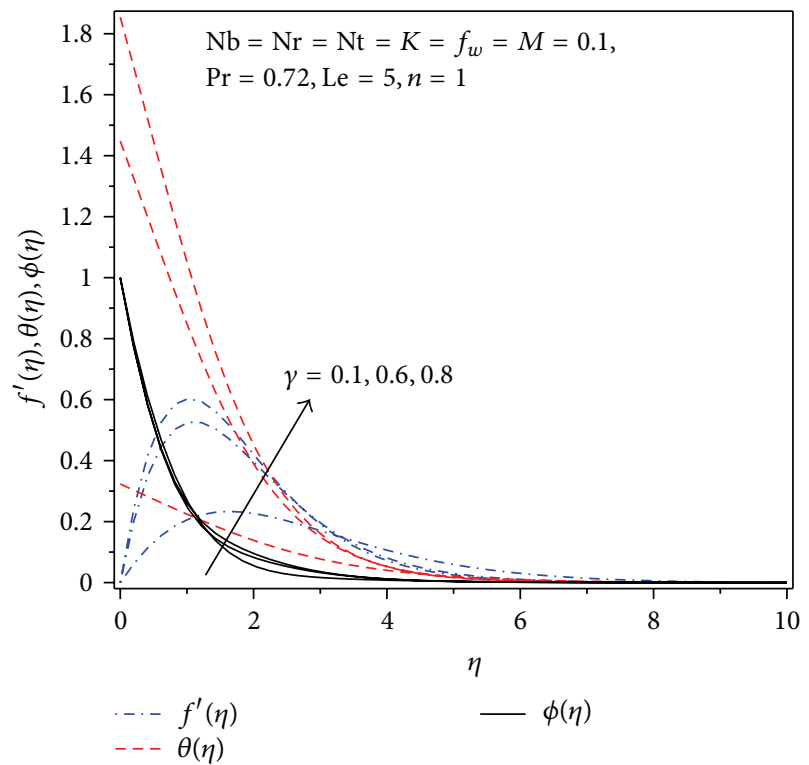

FIGURE 3: Effects of convection-conduction parameter on the dimensionless velocity, temperature, and nanoparticles volume fraction.

\section{Discussion of Results}

Figure 2 shows the flow characteristics for the case studied by Kuznetsov and Nield [23]. The trends are almost identical to those computed by these researchers. The effects of different thermophysical parameters on the dimensionless velocity, temperature, and the nanoparticle volume fraction distributions are illustrated in Figures 3-10. In all the plots suction is present at the sheet $\left(f_{w}>0\right)$ unless otherwise indicated. Also generally $\mathrm{Nr}$ is specified less than unity throughout so that thermal buoyancy force always exceeds species buoyancy force. Figure 3 displays the influence of the thermal convective parameter $(\gamma)$ in the presence of

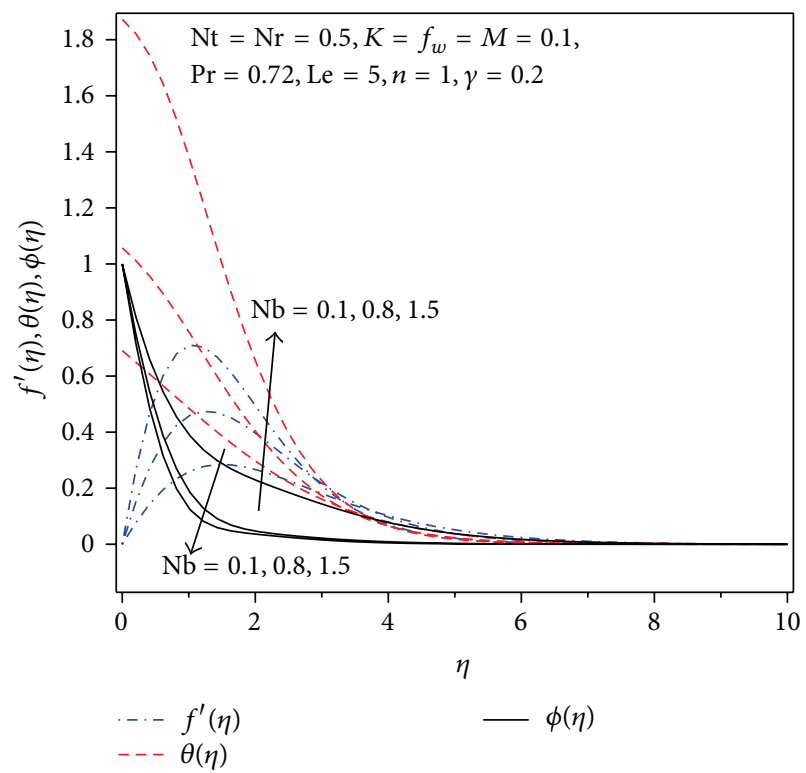

FIgURE 4: Effects of Brownian motion parameter Nb on the dimensionless velocity, temperature, and nanoparticles volume fraction profiles.

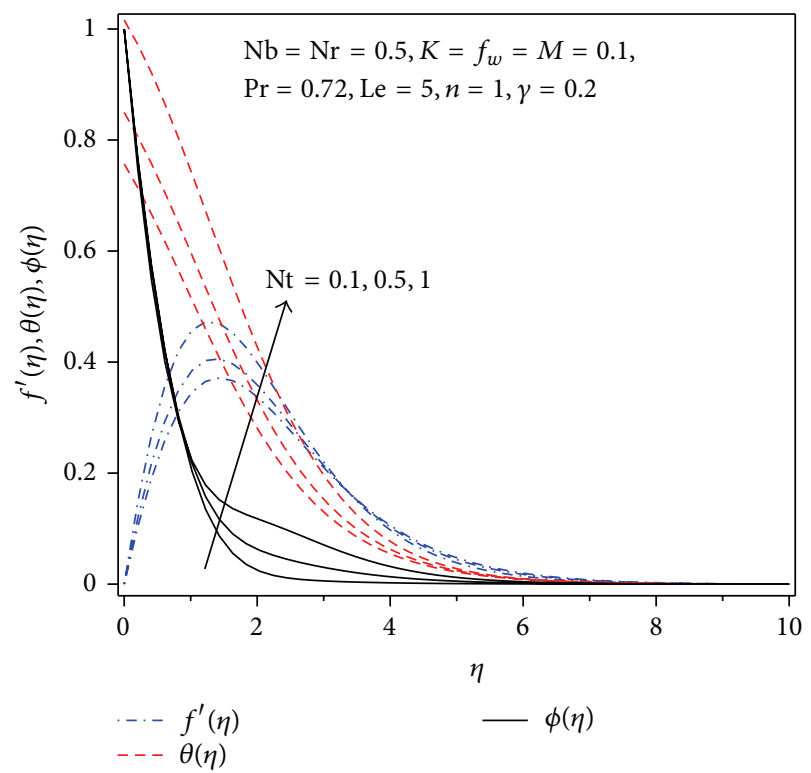

FIGURE 5: Effects of thermophoresis parameter Nt on the dimensionless velocity, temperature, and nanoparticle volume fraction profiles.

uniform (first order) chemical reaction $(n=1)$ and weak magnetic field $(M=0.1)$. The velocity, temperature, and the nanoparticle volume fraction of the fluid all increase with an increase of the strength of $\gamma$. Increasingly stronger thermal convective boundary condition accelerates the flow since the fluid on the right surface of the sheet is heated by the hot fluid on the left surface of the sheet, making it lighter and thus causing it to flow faster. Increasing convective boundary effect (also termed the convection-conduction parameter) heats the boundary layer, as expected, principally at the wall 


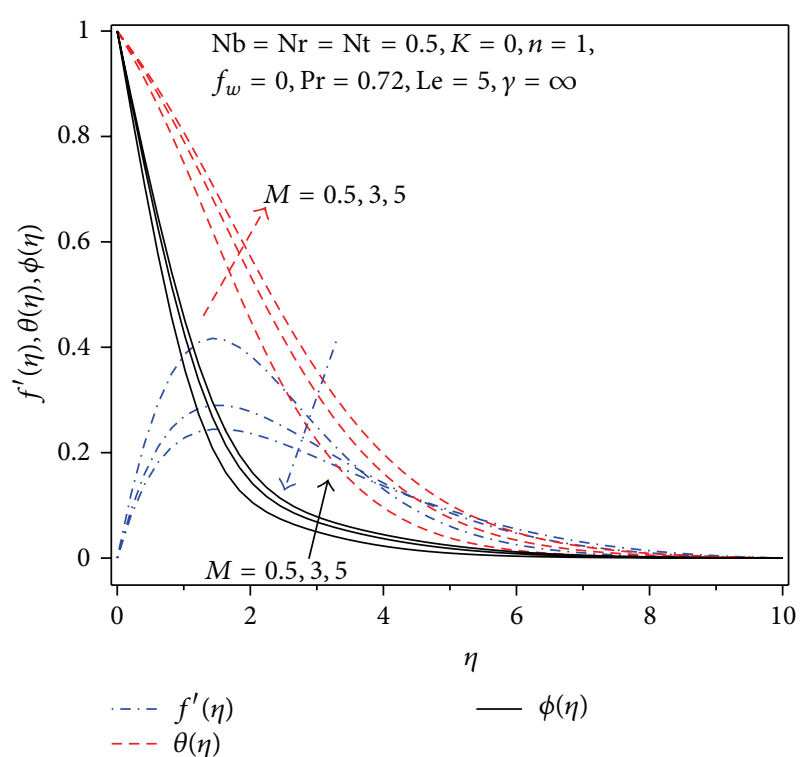

FIGURE 6: Effect of magnetic field parameter $M$ on the dimensionless velocity, temperature, and nanoparticle volume fraction profiles for isothermal plate.

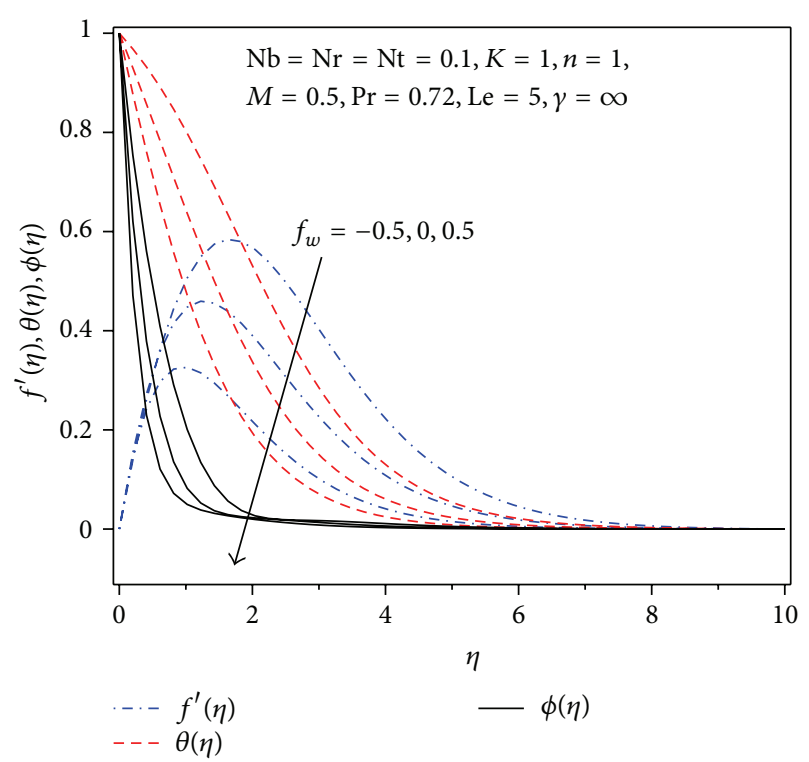

FIGURE 7: Effect of suction/injection parameter $f_{w}$ on the dimensionless velocity, temperature, and nanoparticle volume fraction profiles.

(sheet). The slight increase in nanoparticle volume fraction is localized to a region some distance from the wall. The thermal boundary condition $\theta^{\prime}(0)=-\gamma[1-\theta(0)]$ clearly affects the temperature distribution and this in turn indirectly influences the concentration field (nanoparticle volume fraction) via the coupling terms, $\mathrm{Nb} \theta^{\prime} \phi^{\prime}$ and $(\mathrm{Nt} / \mathrm{Nb}) \theta^{\prime}$. Momentum and thermal boundary layer thicknesses are markedly decreased and increased, respectively, with increasing thermal convective parameter. Figure 4 reveals that with

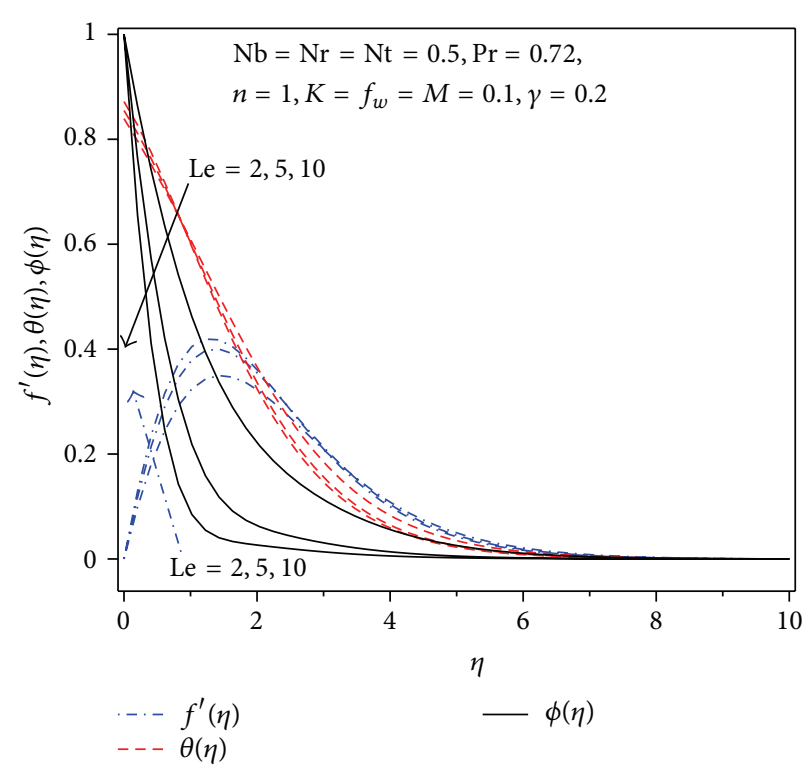

FIGURE 8: Effects of Lewis number Le on the dimensionless velocity, temperature, and nanoparticle volume fraction profiles.

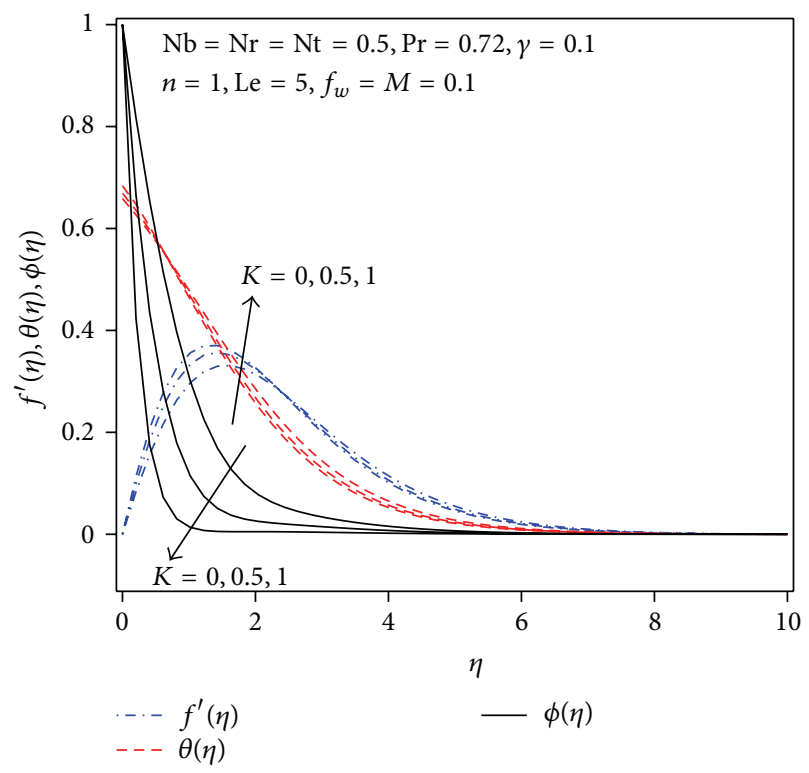

FIGURE 9: Effects of reaction parameter on the dimensionless velocity, temperature, and nanoparticle volume fraction profiles.

increasing Brownian motion parameter, $\mathrm{Nb}$, both dimensionless velocity and temperature are boosted whereas the nanoparticle volume fraction is depressed. Again this figure corresponds to a generative reaction $(K>0)$ of first order $(n=1)$. Thermal enhancement with increasing Brownian motion is clearly demonstrated and thermal boundary layer thickness will also be greater. Many explanations have been provided for this phenomenon including the ballistic transport of energy carriers within individual nanoparticles and between nanoparticles which are in contact and also nanoparticle structuring, as elaborated by Prasher et al. [62]. 


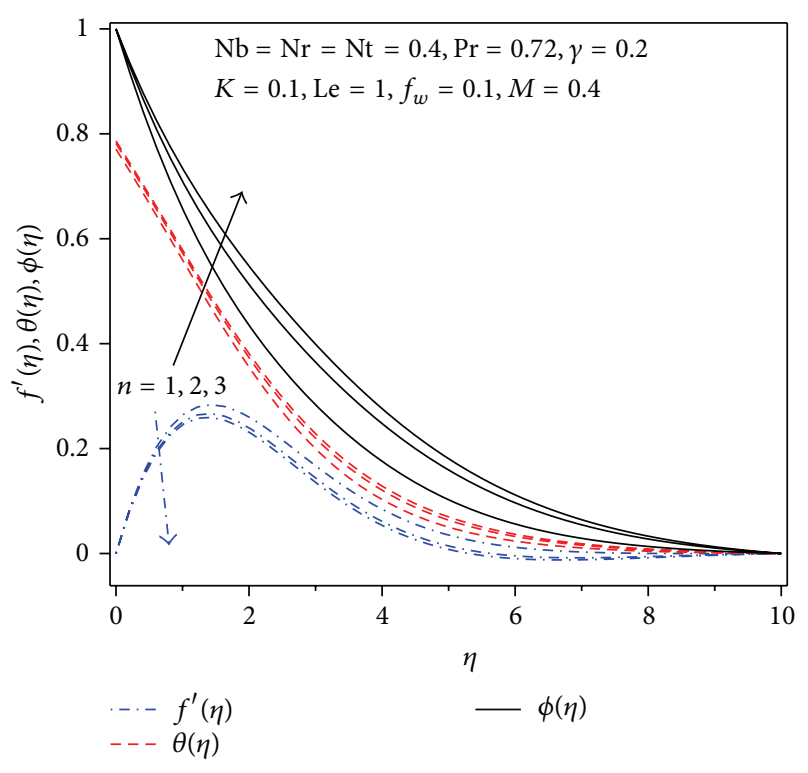

FIGURE 10: Effect of order of chemical reaction $n$ on the dimensionless velocity, temperature, and nanoparticle volume fraction profiles.

For larger diameter nanoparticles, $\mathrm{Nb}$ is small and Brownian motion is weak; the converse is the case for small diameter nanoparticles ( $\mathrm{Nb}$ is high and Brownian motion vigorous). As also observed in Figure 4, increasing $\mathrm{Nb}$ values significantly reduce the nanoparticle volume fraction. This concurs with many other studies of nanofluid transport including Ismoen et al. [40], Makinde and Aziz [28], and Yacob et al. [29]. Figure 5 illustrates that an increase in thermophoresis parameter (Nt) consistently enhances velocity, temperature, and nanoparticle volume fraction of the nanofluid. With greater $\mathrm{Nt}$ values the nanoparticle deposition away from the plate is encouraged. Species diffusion in the boundary layer regime is therefore assisted as is thermal (energy) diffusion. Both effects aid in momentum development leading to a reduction in velocity boundary layer thickness with acceleration in the flow. The velocity peak near the sheet is again witnessed and the pattern is similar to that reported by Chamkha and Aly [25]. We further note that the presence of suction at the sheet $\left(f_{w}>0\right)$ discourages the possibility of flow reversal since it ensures strong adherence of the boundary layer to the wall. Figure 6 shows that an increase in magnetic parameter $(M)$ markedly decelerates the boundary layer flow and that no backflow is generated even in the presence of an impermeable sheet $\left(f_{w}=0\right.$, i.e., solid wall). The transverse magnetic field produces a Lorentzian magnetohydrodynamic drag force perpendicular to the direction of the applied magnetic field, that is, antiparallel to the free stream. This inhibits momentum development and retards the flow, increasing momentum boundary layer thickness. Conversely the temperature is found to be noticeably elevated with increasing $M$ values. Supplementary work has to be expended in dragging the fluid against the action of the magnetic field and this is expended as thermal energy, heating the regime. Thermal boundary layer thickness is therefore enhanced with increasing magnetic field. The assistive effect of magnetohydrodynamics body force on the thermal diffusion also acts to promote species diffusion leading to an elevation in nanoparticle volume fraction values. Stronger magnetic field therefore regulates the flow field but boosts thermal and species diffusion. However the magnitudes of temperature are evidently much greater than nanoparticle volume fraction at all locations in the boundary layer, transverse to the sheet. It is also pertinent to note that the present simulations are valid for small magnetic Reynolds numbers (which relates the ratio of the fluid flux to the magnetic diffusivity) is not adequately large for the magnetic field to be distorted by the flow. For cases where there is a large magnetic Reynolds number, magnetic induction effects must be considered and these are presently under investigation [63]. Figure 7 indicates that velocity is depressed (all profiles decrease monotonically) with an increase in suction parameter $\left(f_{w}>0\right.$, i.e., lateral mass flux through the sheet from the fluid regime). Suction therefore thickens the momentum boundary layer since it decelerates the flow, stabilizes the boundary layer growth, and prevents boundary layer separation. Similarly, temperature and nanoparticle volume fraction values also decrease continuously with an increase of suction parameter $f_{w}$. The opposite behavior is observed for increasing injection $\left(f_{w}<\right.$ 0 ). With injection, more nanofluid is blown through the sheet pores and this accelerates the flow leading to a decrease in momentum boundary layer thickness. However thermal and concentration boundary layer thicknesses will be elevated with injection. Figure 8 illustrates that an increase in Lewis number enhances velocity whereas it causes a decrease in temperature and nanoparticle volume fraction. As featured in other plots, there is also a faster decay of nanoparticle volume fraction profiles from the maximum values at the sheet than there is with temperature profiles. Lewis number is defined to be the ratio of thermal diffusivity to mass (nanoparticle species) diffusivity and characterizes transport phenomena where there were simultaneous heat and mass transfer by convection. The Lewis number also represents the ratio of the Schmidt number to the Prandtl number. When Lewis number is unity, thermal and nanoparticle volume fraction (species) boundary layer thicknesses are equal as are the thermal and species diffusion rates. For values of Lewis number greater than unity, thermal diffusion is faster than species diffusion and species (concentration) boundary layer thickness is exceeded by thermal boundary layer thickness. Thus, the nanoparticle volume fraction magnitudes are expected to decrease with increasing Lewis number. Furthermore, a greater value of Lewis number implies a lower Brownian motion coefficient, $D_{B}$, for a base fluid having kinematic viscosity, $\nu$. Consequently, the higher Lewis number reduces concentration and its boundary layer thickness. Figure 9 demonstrates that with increasing values of chemical reaction parameter, $K$, the nanoparticle volume fraction is strongly reduced whereas the velocity and temperature are slightly enhanced. With greater values of $K$, the nanoparticles are destroyed in concentration, via chemical reaction and this suppresses nanoparticle diffusion rates. Nanoparticle volume fraction (species) boundary layer thickness will therefore be increased. The increasing effect of chemical reaction also 
adds thermal energy to the flow and this heats the boundary layer and accelerates the flow. Effectively nanoparticle volume fraction is a maximum in the absence of chemical reaction; that is, $K=0$, for which the reactive term, $\operatorname{Le} K \phi^{n}$, vanishes in (15). Finally Figure 10 implies that with an increase in the order of the chemical reaction parameter, $n$, there is a corresponding decrease in velocity. The flow is therefore decelerated as the nature of the chemical reaction alters from first order to second and then third order. Conversely there is a little increase in temperatures and nanoparticle volume fractions with greater order of chemical reaction. Higher order chemical reactions of the homogenous type would therefore appear to assist in thermal and species diffusion whereas increasing chemical reaction rates (higher $K$ ) have the adverse influence.

\section{Conclusions}

In the current study a mathematical model has been presented for chemically reactive magneto-nanofluid transport phenomena from a vertical plate in the presence of buoyancy forces and wall mass flux effects utilizing the Aziz convective boundary condition. The nonlinear boundary value problem has been transformed into a dimensionless seventh-order ordinary differential equation system via Lie group theoretical methods and solved using MAPLE 17 quadrature routines. Excellent validation of solutions has been demonstrated with previous studies and convergence of solutions has been properly achieved. The present computations have shown the following.

(i) Dimensionless velocity is depressed whereas the temperature and the nanoparticle volume fraction are increased with an increase in magnetohydrodynamic parameter, $M$.

(ii) Dimensionless temperature is enhanced slightly, and nanoparticle volume fraction is elevated whereas velocity is reduced with a rise in the order of chemical reaction, $n$.

(iii) Dimensionless velocity is enhanced whereas temperature and concentration are suppressed with an increase in Lewis number, Le.

(iv) Dimensionless velocity, temperature, and nanoparticle volume fraction are increased with a rise in injection (wall blowing, i.e., $f_{w}<0$ ) parameter, whereas they are decreased with greater suction $\left(f_{w}>\right.$ $0)$.

(v) Dimensionless velocity is boosted and temperature is enhanced slightly whereas nanoparticle volume fraction is reduced with increasing chemical reaction parameter, $K$.

(vi) Dimensionless velocity and temperature are boosted whereas the nanoparticle volume fraction is depressed with increasing Brownian motion parameter, $\mathrm{Nb}$. (vii) Dimensionless velocity, temperature, and nanoparticle volume fraction are elevated with an increase in thermophoresis parameter, Nt.

(viii) Dimensionless velocity, temperature, and nanoparticle volume fraction are enhanced with increasing convective boundary parameter, $\gamma$.

The present study has considered purely fluid media and magnetic field effects. Future studies will consider electrical field aspects [63] and also permeable media flows [64] for the case of nanofluids.

\section{Conflict of Interests}

The authors declare that there is no conflict of interests regarding the publication of this paper.

\section{References}

[1] S. G. Etemad, B. Farajollahi, M. Hajipour, and J. Thibault, "Turbulent convective heat transfer of suspensions of $\Gamma-\mathrm{AL}_{2} \mathrm{O}_{3}$ and cuonanoparticles (nanofluids)," Journal of Enhanced Heat Transfer, vol. 19, no. 3, pp. 191-197, 2012.

[2] O. A. Bég and D. Tripathi, "Mathematica simulation of peristaltic pumping with double-diffusive convection in nanofluids: a bio-nano-engineering model," Journal of Nanoengineering and Nanosystems, vol. 225, no. 3, pp. 99-114, 2012.

[3] O. A. Bég, T. A. Bég, M. M. Rashidi, and M. Asadi, "DTMPadé semi numerical simulation of nanofluid transport in porous media," International Journal of Applied Mathematics and Mechanics, vol. 9, pp. 80-107, 2013.

[4] P. Rana, R. Bhargava, and O. A. Bég, "Numerical solution for mixed convection boundary layer flow of a nanofluid along an inclined plate embedded in a porous medium," Computers and Mathematics with Applications, vol. 64, no. 9, pp. 2816-2832, 2012.

[5] O. A. Bég, T. A. Bég, M. M. Rashidi, and M. Asadi, "Homotopy semi-numerical modelling of nanofluid convection boundary layers from an isothermal spherical body in a permeable regime," International Journal of Microscale and Nanoscale Thermal Fluid Transport Phenomena, vol. 3, pp. 237-266, 2012.

[6] M. M. Rashidi, O. A. Bég, N. Freidooni Mehr, A. Hosseini, and R. S. Gorla, "Homotopy simulation of axisymmetric laminar mixed convection nanofluid boundary layer flow over a vertical cylinder," Theoretical and Applied Mechanics, vol. 39, no. 4, pp. 365-390, 2012.

[7] K. Jayaraman, K. V. Anand, S. R. Chakravarthy, and R. Sarathi, "Effect of nano-aluminium in plateau-burning and catalyzed composite solid propellant combustion," Combustion and Flame, vol. 156, no. 8, pp. 1662-1673, 2009.

[8] P. Esmaeilzadeh, Z. Fakhroueian, and A. A. Miran Beigi, "Synthesis of biopolymeric $\alpha$-lactalbumin protein nanoparticles and nanospheres as green nanofluids using in drug delivery and food technology," Journal of Nano Research, vol. 16, pp. 89-96, 2012.

[9] M. Muthtamilselvan and R. Rakkiyappan, "Mixed convection in a lid-driven square cavity filled with nanofluids," International Journal of Nanomechanics Science and Technology, vol. 2, no. 4, pp. 275-294, 2011.

[10] M. J. Uddin, N. H. M. Yusoff, O. A. Bég, and A. I. Ismail, "Lie group analysis and numerical solutions for non-Newtonian 
nanofluid flow in a porous medium with internal heat generation," Physica Scripta, vol. 87, no. 2, Article ID 025401, 2013.

[11] K. Y. Leong, R. Saidur, S. N. Kazi, and A. H. Mamun, "Performance investigation of an automotive car radiator operated with nanofluid-based coolants (nanofluid as a coolant in a radiator)," Applied Thermal Engineering, vol. 30, no. 17-18, pp. 2685-2692, 2010.

[12] S. M. S. Murshed, K. C. Leong, and C. Yang, “Thermophysical and electrokinetic properties of nanofluids-a critical review," Applied Thermal Engineering, vol. 28, no. 17-18, pp. 2109-2125, 2008.

[13] S. Özerinç, S. Kakaç, and A. Yazicioǧlu, "Enhanced thermal conductivity of nanofluids: a state-of-the-art review," Microfluidics and Nanofluidics, vol. 8, no. 2, pp. 145-170, 2010.

[14] S. U. S. Choi, "Nanofluids: from vision to reality through research," Journal of Heat Transfer, vol. 131, no. 3, pp. 1-9, 2009.

[15] K. V. Wong and O. Leon, "Applications of nanofluids: current and future," Advances in Mechanical Engineering, vol. 2010, Article ID 519659, 10 pages, 2010.

[16] K. Parekh and H. S. Lee, "Experimental investigation of thermal conductivity of magnetic nanofluids," AIP Conference Proceeding, vol. 1447, pp. 385-386, 2011.

[17] E. Andablo-Reyes, R. Hidalgo-Álvarez, and J. de Vicente, "Controlling friction using magnetic nanofluids," Soft Matter, vol. 7, no. 3, pp. 880-883, 2011.

[18] Y.-C. Chiang, J.-J. Chieh, and C.-C. Ho, "The magneticnanofluid heat pipe with superior thermal properties through magnetic enhancement," Nanoscale Research Letters, vol. 7, article 322, 2012.

[19] R. Patel, "Effective viscosity of magnetic nanofluids through capillaries," Physical Review E-Statistical, Nonlinear, and Soft Matter Physics, vol. 85, no. 2, Article ID 026316, 2012.

[20] P. Rana, R. Bhargava, and O. A. Bég, "Finite element simulation of unsteady magneto-hydrodynamic transport phenomena on a stretching sheet in a rotating nanofluid," Proceedings of the Institution of Mechanical Engineers. Part N: Journal of Nanoengineering and Nanosystems, vol. 227, no. 2, pp. 77-99, 2013.

[21] D. Yadav, R. Bhargava, and G. S. Agrawal, "Thermal instability in a nanofluid layer with a vertical magnetic field," Journal of Engineering Mathematics, vol. 80, pp. 147-164, 2013.

[22] J. Buongiorno, “Convective transport in nanofluids," Journal of Heat Transfer, vol. 128, no. 3, pp. 240-250, 2006.

[23] A. V. Kuznetsov and D. A. Nield, "Natural convective boundarylayer flow of a nanofluid past a vertical plate," International Journal of Thermal Sciences, vol. 49, no. 2, pp. 243-247, 2010.

[24] M. A. Hamad, I. Pop, and A. I. Ismail, "Magnetic field effects on free convection flow of a nanofluid past a vertical semi-infinite flat plate," Nonlinear Analysis: Real World Applications, vol. 12, no. 3, pp. 1338-1346, 2011.

[25] A. J. Chamkha and A. M. Aly, "MHD free convection flow of a nanofluid past a vertical plate in the presence of heat generation or absorption effects," Chemical Engineering Communications, vol. 198, no. 3, pp. 425-441, 2011.

[26] A. Aziz, "Similarity solution for laminar thermal boundary over a flat plate with a convective boundary condition," Communication in Nonlinear Science and Numerical Simulation, vol. 14, pp. 1064-1068, 2009.

[27] S. Yao, T. Fang, and Y. Zhong, "Heat transfer of a generalized stretching/shrinking wall problem with convective boundary conditions," Communications in Nonlinear Science and Numerical Simulation, vol. 16, no. 2, pp. 752-760, 2011.
[28] O. D. Makinde and A. Aziz, "Boundary layer flow of a nanofluid past a stretching sheet with a convective boundary condition," International Journal of Thermal Sciences, vol. 50, no. 7, pp. 13261332, 2011.

[29] N. A. Yacob, A. Ishak, I. Pop, and K. Vajravelu, "Boundary layer flow past a stretching/shrinking surface beneath an external uniform shear flow with a convective surface boundary condition in a nanofluid," Nanoscale Research Letters, vol. 6, no. 1, pp. 314-320, 2011.

[30] M. J. Uddin, W. A. Khan, and A. I. Ismail, "Free convection boundary layer flow from a heated upward facing horizontal flat plate embedded in a porous medium filled by a nanofluid with convective boundary condition," Transport in Porous Media, vol. 92, no. 3, pp. 867-881, 2012.

[31] A. Aziz and W. A. Khan, "Natural convective boundary layer flow of a nanofluid past a convectively heated vertical plate," International Journal of Thermal Sciences, vol. 52, no. 1, pp. 8390, 2012.

[32] M. A. Chaudhary and J. H. Merkin, "A simple isothermal model for homogeneous-heterogeneous reactions in boundary-layer flow. I. Equal diffusivities," Fluid Dynamics Research, vol. 16, no. 6, pp. 311-333, 1995.

[33] O. A. Bég, M. M. Rashidi, M. Keimanesh, and T. A. Bég, "Semi-numerical modelling of 'chemically-frozen' combusting buoyancy-driven boundary layer flow along an inclined surface," International Journal of Applied Mathematics Mechanics, vol. 9, pp. 1-16, 2013.

[34] J. H. Merkin and T. Mahmood, "The convective flow on a reacting surface in a porous medium," Transport in Porous Media, vol. 33, no. 3, pp. 279-293, 1998.

[35] O. A. Bég, R. Bhargava, S. Rawat, H. S. Takhar, and T. A. Bég, "A study of steady buoyancy-driven dissipative micropolar free convection heat and mass transfer in a Darcian porous regime with chemical reaction," Nonlinear Analysis. Modelling and Control, vol. 12, no. 2, pp. 157-180, 2007.

[36] E.-S. Chia, A chemical reaction engineering perspective of polymer electrolyte membrane fuel cells [Ph.D. thesis], Chemical Engineering, Princeton University, Princeton, NJ, USA, 2006.

[37] S. Steinfeld and R. Palumbo, "Solar thermochemical process technology," in Encyclopedia of Physical Science \& Technology, R. A. Meyers, Ed., vol. 15, pp. 237-256, Academic Press, 2001.

[38] O. D. Makinde, K. Zimba, and O. A. Bég, "Numerical study of chemically reacting hydromagnetic boundary layer flow with Soret/Dufour effects and a convective surface boundary condition," International Journal of Thermal and Environmental Engineering, vol. 4, no. 1, pp. 89-98, 2012.

[39] L. Chen, H. Xie, Y. Li, and W. Yu, "Nanofluids containing carbon nanotubes treated by mechanochemical reaction," Thermochimica Acta, vol. 477, no. 1-2, pp. 21-24, 2008.

[40] M. Ismoen, R. Kandasamy, and R. Abdul Kahar, "Scaling group transformation forboundary-layer flow of a nanofluid past a porous vertical stretching surface in the presence of chemical reaction with heat radiation," Computers and Fluids, vol. 52, pp. 15-21, 2011.

[41] P. K. Kameswaran, M. Narayana, P. Sibanda, and P. V. S. N. Murthy, "Hydromagnetic nanofluid flow due to a stretching or shrinking sheet with viscous dissipation and chemical reaction effects," International Journal of Heat and Mass Transfer, vol. 55, no. 25-26, pp. 7587-7595, 2012.

[42] N. C. Rosca, T. Grosan, and I. Pop, "Stagnation-point flow and mass transfer with chemical reaction past a permeable 
stretching/shrinking sheet in a nanofluid," Sains Malaysiana, vol. 41, no. 10, pp. 1271-1279, 2012.

[43] R. K. Tiwari and M. K. Das, "Heat transfer augmentation in a two-sided lid-driven differentially heated square cavity utilizing nanofluids," International Journal of Heat and Mass Transfer, vol. 50, no. 9-10, pp. 2002-2018, 2007.

[44] S. Samanta and A. Guha, "Analysis of heat transfer and stability of magnetohydrodynamic natural convection above a horizontal plate with heat flux boundary condition," International Journal of Heat and Mass Transfer, vol. 70, pp. 793-802, 2014.

[45] M. J. Uddin, O. A. Bég, and N. Amin, "Hydromagnetic transport phenomena from a stretching or shrinking nonlinear nanomaterial sheet with Navier slip and convective heating: a model for bio-nano-materials processing," Journal of Magnetism and Magnetic Materials, vol. 368, pp. 252-261, 2014.

[46] K. R. Khair and A. Bejan, "Mass transfer to natural convection boundary layer flow driven by heat transfer," Journal of Heat Transfer, vol. 107, no. 4, pp. 979-981, 1985.

[47] P. K. Ma and W. H. Hui, "Similarity solutions of the twodimensional unsteady boundary-layer equations," Journal of Fluid Mechanics, vol. 216, pp. 537-559, 1990.

[48] R. Seshadri and T. Y. Na, Group Invariance in Engineering Boundary Value Problems, Springer, New York, NY, USA, 1985.

[49] J. M. Hill, Differential Equations and Group Methods for Scientists and Engineers, CRC Press, Boca Raton, Fla, USA, 1992.

[50] R. E. White and V. R. Subramanian, Computational Methods in Chemical Engineering with Maple, Springer, New York, NY, USA, 2010.

[51] K. Atalık, "Group theoretical analysis and similarity solutions for stress boundary layers in viscoelastic flows," Journal of NonNewtonian Fluid Mechanics, vol. 153, no. 1, pp. 62-71, 2008.

[52] V. N. Grebenev, M. Oberlack, and A. N. Grishkov, "Lie algebra methods for the applications to the statistical theory of turbulence," Journal of Nonlinear Mathematical Physics, vol. 15, no. 2, pp. 227-251, 2008.

[53] M. M. Rashidi, N. Rahimzadeh, M. Ferdows, J. Uddin, and O. A. Bég, "Group theory and differential transform analysis of mixed convective heat and mass transfer from a horizontal surface with chemical reaction effects," Chemical Engineering Communications, vol. 199, no. 8, pp. 1012-1043, 2012.

[54] P. P. Arasu, P. Loganathan, R. Kandasamy, and I. Muhaimin, "Lie group analysis for thermal-diffusion and diffusion-thermo effects on free convective flow over a porous stretching surface with variable stream conditions in the presence of thermophoresis particle deposition," Nonlinear Analysis. Hybrid Systems, vol. 5, no. 1, pp. 20-31, 2011.

[55] Y. Z. Boutros, M. B. Abd-El-Malek, N. A. Badran, and H. S. Hassan, "Lie-group method of solution for steady twodimensional boundary-layer stagnation-point flow towards a heated stretching sheet placed in a porous medium," Meccanica, vol. 41, no. 6, pp. 681-691, 2006.

[56] S. Mukhopadhyay and G. C. Layek, "Effects of variable fluid viscosity on flow past a heated stretching sheet embedded in a porous medium in presence of heat source/sink," Meccanica, vol. 47, no. 4, pp. 863-876, 2012.

[57] R. Kandasamy, P. Loganathan, and P. P. P. Arasu, "Scaling group transformation for MHD boundary-layer flow of a nanofluid past a vertical stretching surface in the presence of suction/injection," Nuclear Engineering and Design, vol. 241, no. 6, pp. 2053-2059, 2011.
[58] M. A. A. Hamad, M. J. Uddin, and A. I. M. Ismail, "Radiation effects on heat and mass transfer in MHD stagnation-point flow over a permeable flat plate with thermal convective surface boundary condition, temperature dependent viscosity and thermal conductivity," Nuclear Engineering and Design, vol. 242, pp. 194-200, 2012.

[59] M. Ferdows, M. J. Uddin, and A. A. Afify, "Scaling group transformation for MHD boundary layer free convective heat and mass transfer flow past a convectively heated nonlinear radiating stretching sheet," International Journal of Heat and Mass Transfer, vol. 56, no. 1-2, pp. 181-187, 2013.

[60] O. A. Bég, "Numerical methods for multi-physical magnetohydrodynamics," in New Developments in Hydrodynamics Research, M. J. Ibragimov and M. A. Anisimov, Eds., pp. 1-112, Nova Science, New York, NY, USA, 2012.

[61] A. Pantokratoras, "A common error made in investigation of boundary layer flows," Applied Mathematical Modelling, vol. 33, no. 1, pp. 413-422, 2009.

[62] R. Prasher, P. Bhattacharya, and P. E. Phelan, "Thermal conductivity of nanoscale colloidal solutions (Nanofluids)," Physical Review Letters, vol. 94, no. 2, pp. 25901-1-25901-4, 2005.

[63] O. A. Bég, M. Hameed, and T. A. Bég, "Chebyshev spectral collocation simulation of nonlinear boundary value problems in electrohydrodynamics," International Journal for Computational Methods in Engineering Science and Mechanics, vol. 14, no. 2, pp. 104-115, 2013.

[64] O. A. Bég, J. Zueco, R. Bhargava, and H. S. Takhar, "Magnetohydrodynamic convection flow from a sphere to a nonDarcian porous medium with heat generation or absorption effects: network simulation," International Journal of Thermal Sciences, vol. 48, no. 5, pp. 913-921, 2009. 


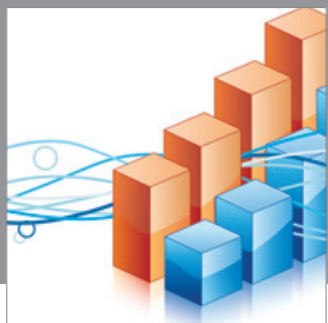

Advances in

Operations Research

mansans

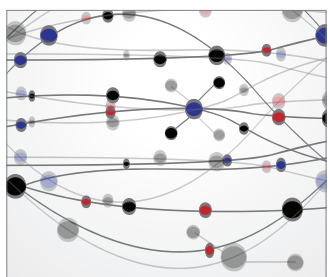

The Scientific World Journal
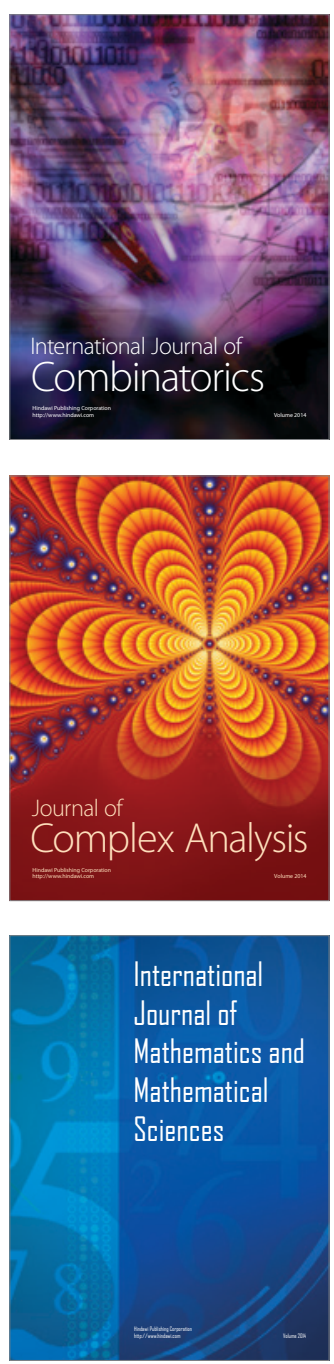
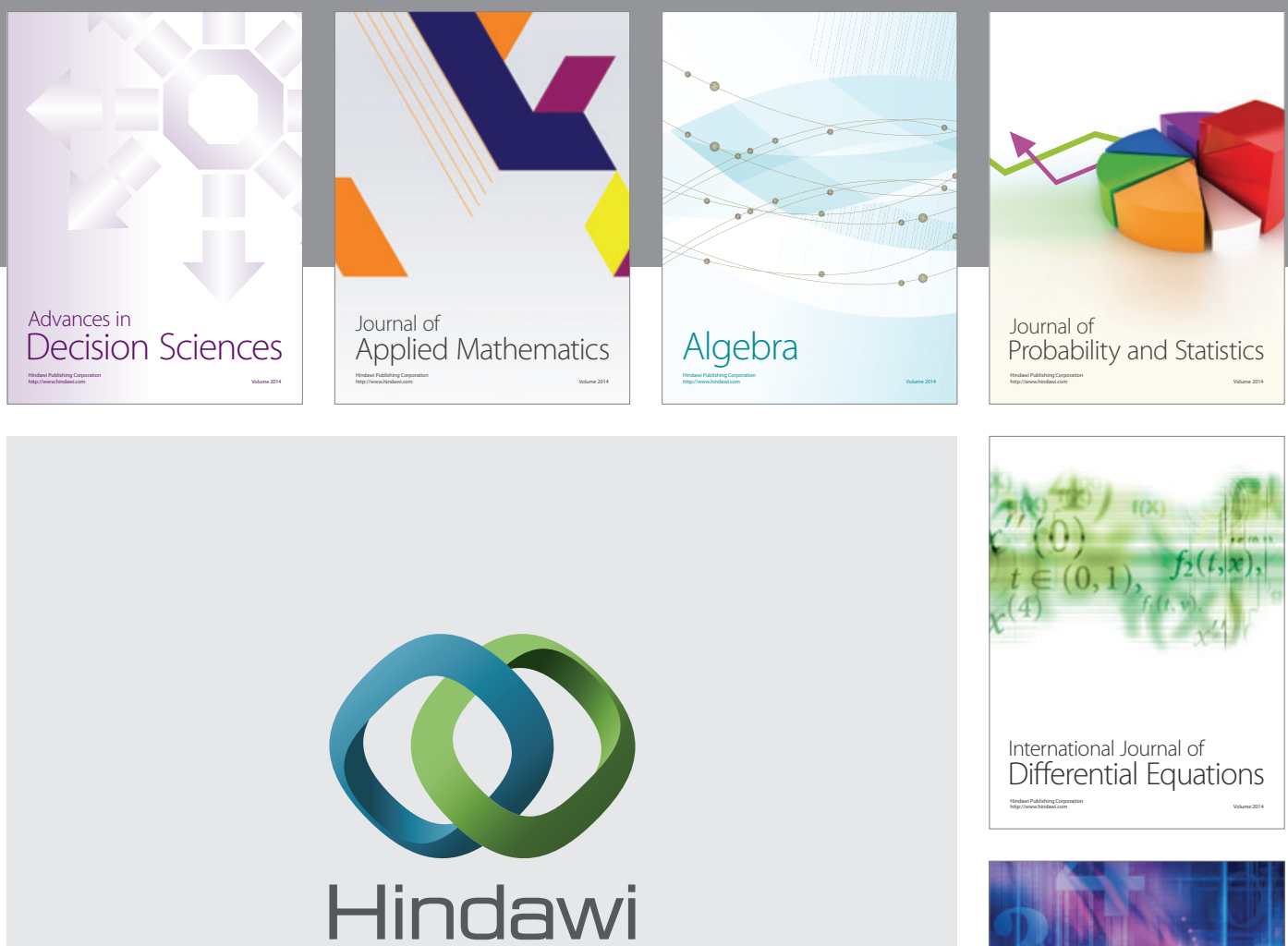

Submit your manuscripts at http://www.hindawi.com
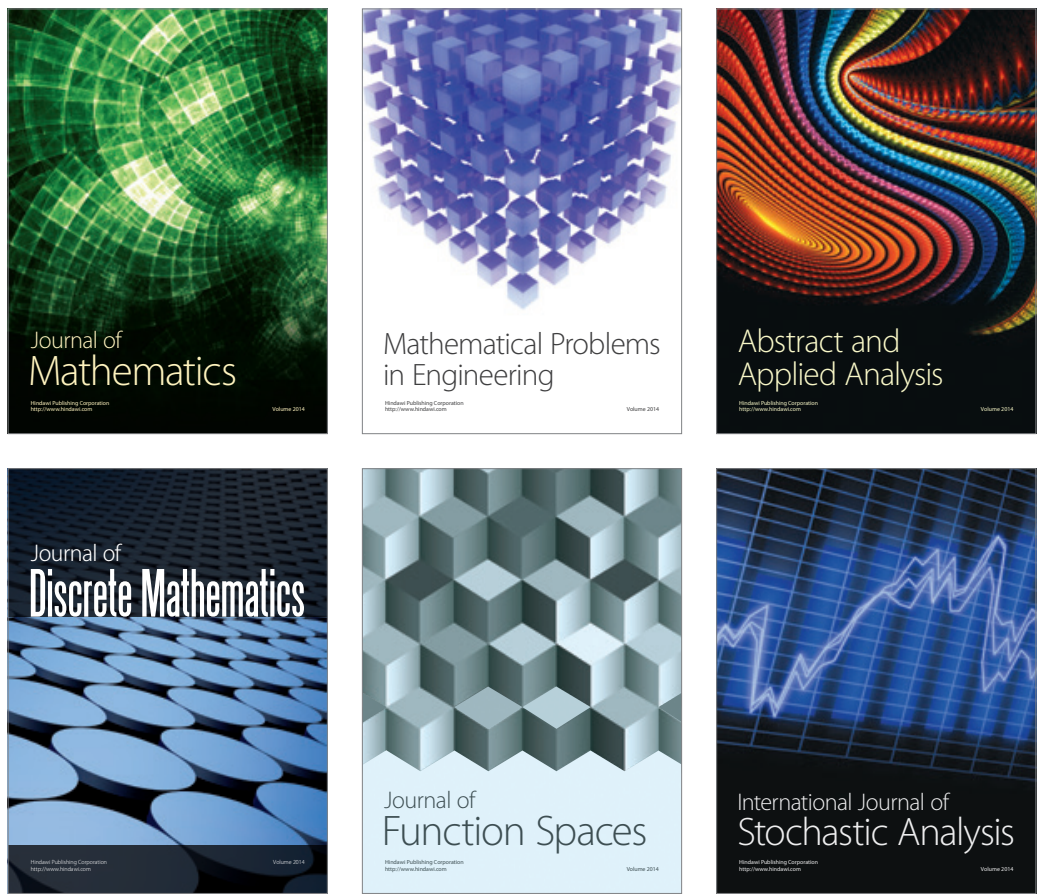

Journal of

Function Spaces

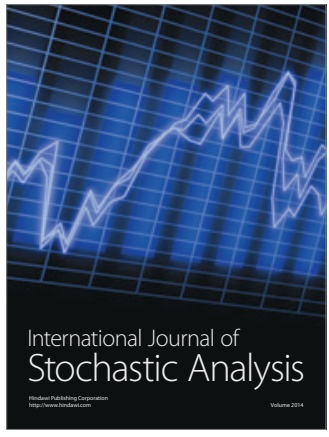

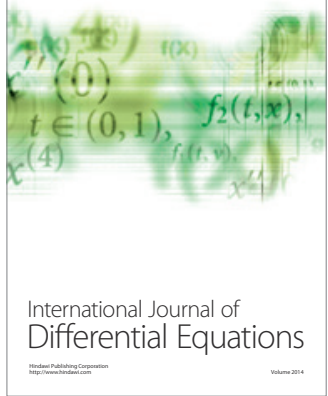
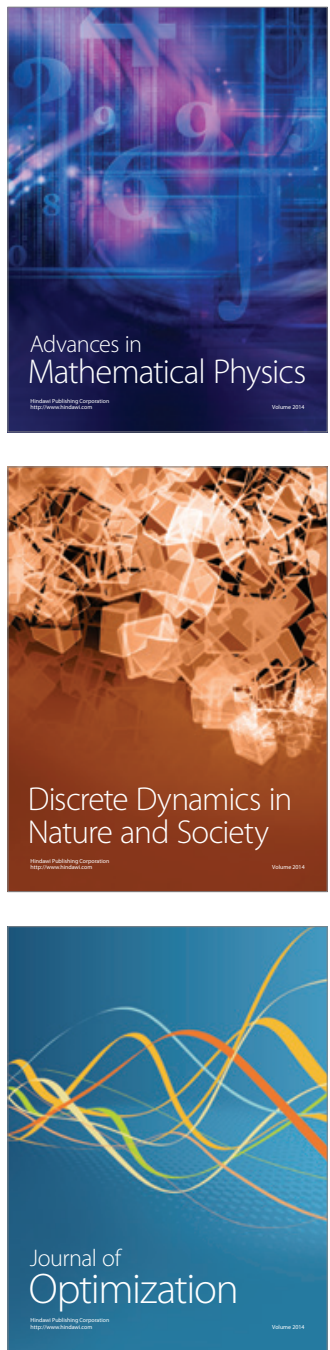